\title{
Normalization of D-instanton amplitudes
}

\author{
Ashoke Sen \\ Harish-Chandra Research Institute, HBNI, \\ Chhatnag Road, Jhusi, Allahabad 211019, India \\ E-mail: sen@hri.res.in
}

\begin{abstract}
D-instanton amplitudes suffer from various infrared divergences associated with tachyonic or massless open string modes, leading to ambiguous contribution to string amplitudes. It has been shown previously that string field theory can resolve these ambiguities and lead to unambiguous expressions for D-instanton contributions to string amplitudes, except for an overall normalization constant that remains undetermined. In this paper we show that string field theory, together with the world-sheet description of the amplitudes, can also fix this normalization constant. We apply our analysis to the special case of two dimensional string theory, obtaining results in agreement with the matrix model results obtained by Balthazar, Rodriguez and Yin.
\end{abstract}

KeYwORDS: D-branes, String Field Theory

ARXIV EPRINT: 2101.08566 


\section{Contents}

1 Introduction 1

2 The normalization constant from the Siegel gauge path integral 2

3 'Gauge invariant' path integral 5

4 The multiplier $\quad 10$

$\begin{array}{lll}5 & \text { Discussion } & 11\end{array}$

A Siegel gauge fixing in the Faddeev-Popov formalism 12

\section{Introduction}

D-instantons give a class of non-perturbative contributions to string amplitudes. One characteristic of these contributions is the presence of an overall multiplicative factor $e^{-C / g_{s}}$ where $g_{s}$ is the closed string coupling and $C$ is a constant. Besides this factor, the amplitudes admit usual perturbation expansion in powers of $g_{s}$. The contribution to an amplitude at any given order in $g_{s}$ can be computed using the standard world-sheet approach by including Riemann surfaces with boundaries ending on the D-instanton, but at each order one encounters certain infra-red divergences [1-3] that render the amplitudes ambiguous. At any given order, these ambiguities can be encoded in a set of undetermined constants. String field theory [4-9] provides an unambiguous procedure for determining these constants, by identifying the physical origin of these infrared divergences and rectifying them based on this understanding [10-13]. So far this procedure has been applied to two dimensional string theory, for which there is a dual matrix model description that can be used to check the results.

However previous analysis left one constant undetermined - namely the overall normalization of the D-instanton amplitude. Formally this is given by the exponential of the annulus amplitude, with D-instanton boundary condition at the two boundaries and no other vertex operator insertion. However the annulus amplitude is divergent due to the presence of massless and tachyonic open string modes on the D-instanton. In conventional string perturbation theory, such diagrams are part of bubble diagrams and drop out in the computation of physical amplitudes. However for D-instanton amplitudes the situation is somewhat different since the D-instanton contribution to the amplitude has to be first added to the perturbative amplitude and then the sum needs to be divided by the sum of perturbative and D-instanton contribution to bubble diagrams. Therefore the overall normalization is physically relevant, and one expects that it should be possible to compute this in string theory. Since string field theory is capable of making sense of infrared 
divergences in the amplitudes, the natural expectation would be that string field theory should be able to give an unambiguous result for the normalization constant. However, when one tries to compute this using string field theory, which in this case is a theory of open and closed strings, one finds that there is no internal consistency requirement within string field theory that can be used to fix this normalization, since this can be changed by adding a field independent constant to the string field theory action that does not violate any constraint coming from the requirement of gauge invariance.

To overcome this problem, we shall take the viewpoint that the world-sheet approach already fixes the normalization as the exponential of the annulus partition function, and the job of string field theory is to simply give physical interpretation of the divergences of the amplitude and render them finite based on this interpretation. We show that the world-sheet result may be regarded as the gauge fixed version of a path integral in string theory with a specific normalization, and the divergences that we encounter arise due to breakdown of the gauge choice. However the 'gauge invariant' form of the path integral, expressed as an integral over the full classical string field divided by the volume of the gauge group, yields unambiguous result. We apply this procedure to the case of two dimensional string theory, and find that the normalization of the one instanton amplitude determined this way agrees with the results of the matrix model computed in [3] following the general formalism developed in [14].

The rest of the paper is organized as follows. In section 2 we express the exponential of the annulus partition function as a path integral over string fields in the Siegel gauge. At this stage the path integral remains singular due to the existence of zero modes, reflecting the singularity of the annulus partition function. In section 3 we trace these singularities to the breakdown of the Siegel gauge, and show that we can get finite result for the path integral by rewriting it in a 'gauge invariant' form. In section 4 we calculate the multiplier factor - the multiple of the steepest descent contour of the D-instanton that forms part of the actual integration contour of the full string theory, and show that after multiplying the exponential of the annulus partition function, computed in section 3 , by this factor, we get agreement with the matrix model result. In section 5 we discuss possible application of our analysis to other systems. In appendix A we show how the central result used in our analysis - the equivalence of the gauge invariant version of the path integral over string fields and the Siegel gauge fixed version of the same path integral, can be proved directly using the standard Faddeev-Popov approach instead of the abstract results of the Batalin-Vilkovisky (BV) formalism [15-21].

\section{The normalization constant from the Siegel gauge path integral}

Our goal is to compute the normalization constant $\mathbf{N}$ appearing in the D-instanton amplitudes. In the world-sheet description it is given by:

$$
\mathbf{N}=\zeta \exp [\mathcal{A}]
$$

where $\mathcal{A}$ is the annulus partition function:

$$
\mathcal{A}=\int_{0}^{\infty} \frac{d t}{2 t} \operatorname{Tr}\left(e^{-2 \pi t L_{0}}\right) .
$$


Here $\operatorname{Tr}$ denotes trace over all states of the open string projected into the Siegel gauge by the projection operator $b_{0} c_{0}$, weighted by $(-1)^{F}$ where $-(-1)^{F}$ denotes the grassmann parity of the vertex operator corresponding to the state. The extra minus sign multiplying $(-1)^{F}$ is a reflection of the fact that bosonic (fermionic) open string modes correspond to grassmann odd (even) states in the world-sheet theory. $\zeta$ in $(2.1)$ is the multiplier factor that depends on how the steepest descent contour associated with the D-instanton fits inside the actual integration contour $[22-24]$. In particular $\exp [\mathcal{A}]$ represents the one loop contribution to the path integral from the full steepest descent contour passing through the instanton solution and $\zeta$ reflects the multiple of the steepest descent contour that forms part of the actual integration contour. We shall see for example that in the two dimensional string theory, $\zeta=1 / 2$ up to a sign. ${ }^{1}$

The constant $\mathbf{N}$ given in (2.1) is the overall multiplicative factor that appears in the instanton induced effective action of the closed string fields [27]. This is related to the normalization constant $\mathcal{N}$ introduced in [3, 28], appearing as a multiplicative factor in the S-matrix, via the relation $\mathcal{N}=i \mathbf{N}$. In our analysis, we shall not be careful in fixing the sign of $\mathbf{N}$, since this will be fixed at the end using separate considerations.

We can express (2.2) as

$$
\mathcal{A}=\int_{0}^{\infty} \frac{d t}{2 t}\left[\sum_{i} e^{-2 \pi t h_{i}^{b}}-\sum_{j} e^{-2 \pi t h_{j}^{f}}\right],
$$

where $\left\{h_{i}^{b}\right\}$ and $\left\{h_{j}^{f}\right\}$ are the $L_{0}$ eigenvalues of the grassmann odd and the grassmann even states of the world-sheet CFT. If we assume that the total number of bosonic modes equals the total number of fermionic modes so that the integrand is finite as $t \rightarrow 0$, and furthermore that the $h_{i}^{b}$ and $h_{j}^{f}$ are positive so that the integrand falls off exponentially as $t \rightarrow \infty$, then the integral (2.3) is finite. In this case it gives the result

$$
\mathcal{A}=\frac{1}{2} \ln \frac{\prod_{j} h_{j}^{f}}{\prod_{i} h_{i}^{b}} .
$$

Substituting this into (2.1), we get

$$
\mathbf{N}=\zeta \sqrt{\frac{\prod_{j} h_{j}^{f}}{\prod_{i} h_{i}^{b}}}
$$

In the system that we shall analyze, $h_{j}^{f}$ 's come in pairs of equal values so that we can write this as

$$
\mathbf{N}=\zeta \frac{\prod_{j}^{\prime} h_{j}^{f}}{\sqrt{\prod_{i} h_{i}^{b}}},
$$

where $\prod_{j}^{\prime}$ corresponds to the product running over only one member for each pair.

\footnotetext{
${ }^{1}$ In string field theory, (2.1) may be justified by demanding that at the tachyon vacuum [25] $\mathbf{N}$ must be 1 so that we get the usual perturbative closed string amplitudes. Since the boundary state vanishes at the tachyon vacuum [26], we have $\mathcal{A}=0$ and therefore $e^{\mathcal{A}}=1$. Furthermore it will be seen in section 4 , figure 1 that $\zeta=1$ at the perturbative vacuum. Therefore (2.1) should not have any additional factor.
} 
We can express this as the result of integration over the bosonic variables $b_{i}$ and fermionic variables $f_{j}, \tilde{f}_{j}$ as follows:

$$
\mathbf{N}=\zeta \int \prod_{i} \frac{d b_{i}}{\sqrt{2 \pi}} \prod_{j} d f_{j} d \widetilde{f}_{j} \exp \left[-\frac{1}{2} \sum_{i} h_{i}^{b} b_{i}^{2}+\sum_{j}^{\prime} h_{j}^{f} \widetilde{f}_{j} f_{j}\right]
$$

Equality of (2.1) and (2.7) is an identity when all the $h_{i}^{b}$ 's and $h_{j}^{f}$ 's are positive, but we shall take (2.7) to be the defining expression for $\mathbf{N}$ even when this condition fails. In particular, we shall apply this formalism to D-instanton system for which some of the $L_{0}$ eigenvalues vanish and / or are negative. A justification for this may be given as follows. Instead of studying open strings on a single D-instanton, we can take a system of two Dinstantons separated along the Euclidean time direction and analyze the states of the open string stretched between the pair of D-instantons. In this case $L_{0}$ will get a non-vanishing contribution from the tension of the stretched open string and the manipulations carried out above will be well defined for sufficiently large separation. We can recover the original system of interest by analytic continuation of this result to zero separation and using the fact that in this limit the spectrum of open strings with two ends lying on different Dinstantons coincides with the spectrum of open strings with both ends lying on the same D-instanton. Of course (2.7) is not well defined in this limit due to the appearance of zero eigenvalues in the bosonic and fermionic sectors, and so it does not lead to a finite unambiguous result for $\mathbf{N}$ at this stage. However, we shall see in section 3 that it is possible to trace these zero eigenvalues to singular gauge choice and transform (2.7) to finite, unambiguous result (3.8) using insights from string field theory.

In (2.7), the variables $b_{i}$ may be interpreted as the bosonic open string fields on the D-instanton, the variables $f_{i}, \widetilde{f}_{i}$ may be interpreted as the fermionic open string fields on the D-instanton and the argument of the exponential may be interpreted as the quadratic part of the action of the open string field theory in the Siegel gauge. To see how this arises, we now review some basic aspects of string field theory.

The off-shell open string field describing the degrees of freedom of a D-instanton is taken to be an arbitrary element $|\Psi\rangle$ of $H$ - the vector space of states of the open string, including matter and ghost excitations. Let $\left\{\left|\phi_{r}\right\rangle\right\}$ be the set of basis states in $H$. Then we can expand $|\Psi\rangle \in H$ as

$$
|\Psi\rangle=\sum_{r} \chi^{r}\left|\phi_{r}\right\rangle
$$

$\left\{\chi^{r}\right\}$ 's are the degrees of freedom over which the path integral is to be performed after suitable gauge fixing. Even though we have referred to the $\chi_{r}$ 's as fields, they are actually zero dimensional fields - ordinary variables - since on the D-instanton the open strings do not carry any continuous momentum labels. Therefore it is more appropriate to call them modes. $\chi^{r}$ has even (odd) grassmann parity if the ghost number of $\phi_{r}$ is odd (even). The kinetic term of the BV master action of string field theory takes the form:

$$
S=-\frac{1}{2}\left\langle\Psi\left|Q_{B}\right| \Psi\right\rangle,
$$


where $Q_{B}$ is the world-sheet BRST operator. The minus sign in front of the action is unusual, but has been introduced keeping in mind that we shall be using a convention in which the Euclidean path integral is weighted by $e^{S}$.

In the $\mathrm{BV}$ formalism the open string modes multiplying states of ghost number $\leq 1$ are regarded as fields and the modes multiplying states of ghost number $\geq 2$ are regarded as antifields. If we introduce basis states $\left\{\left|\varphi_{r}\right\rangle\right\}$ in the ghost number $\leq 1$ subspace and $\left\{\left|\varphi^{r}\right\rangle\right\}$ in the ghost number $\geq 2$ subspace such that

$$
\left\langle\varphi^{r} \mid \varphi_{s}\right\rangle=\delta_{s}^{r}=\left\langle\varphi_{s} \mid \varphi^{r}\right\rangle, \quad\left\langle\varphi^{r} \mid \varphi^{s}\right\rangle=0, \quad\left\langle\varphi_{r} \mid \varphi_{s}\right\rangle=0,
$$

and expand the string field as,

$$
|\Psi\rangle=\sum_{r}\left(\psi^{r}\left|\varphi_{r}\right\rangle+\psi_{r}\left|\varphi^{r}\right\rangle\right),
$$

then we call $\psi^{r}$ a field and $\psi_{r}$ the conjugate anti-field up to a sign. The path integral is carried out over a Lagrangian submanifold. For our analysis it will be sufficient to consider a special class of Lagrangian submanifolds in which, for each pair $\left(\psi^{r}, \psi_{r}\right)$, we set either $\psi^{r}$ to 0 or $\psi_{r}$ to 0 . The path integral can be shown to be formally independent of the choice of the Lagrangian submanifold. The Siegel gauge corresponds to the choice of the Lagrangian submanifold in which we impose the condition:

$$
b_{0}|\Psi\rangle=0 .
$$

In this gauge the action (2.9) takes the form:

$$
S_{\text {g.f. }}=-\frac{1}{2}\left\langle\Psi\left|c_{0} L_{0}\right| \Psi\right\rangle \text {. }
$$

If we choose the basis states $\left\{\left|\phi_{r}^{(n)}\right\rangle\right\}$ of ghost number $n$ in the Siegel gauge, satisfying

$$
b_{0}\left|\phi_{r}^{(n)}\right\rangle=0, \quad\left\langle\phi_{r}^{(2-n)}\left|c_{0}\right| \phi_{s}^{(n)}\right\rangle=\delta_{r s} \quad \text { for } n \leq 1,
$$

then by expanding $|\Psi\rangle$ in this basis and substituting in the action (2.13), we recover the exponent in (2.7) if we identify the variables $b_{i}, f_{i}$ and $\tilde{f}_{i}$ as the coefficients of expansion of $|\Psi\rangle$ in this basis.

This shows that (2.7) may be given an interpretation as path integral over the open string fields in the Siegel gauge. Note however that (2.7) comes with a specific normalization of the integration measure that will be important for us. String field theory, by itself, cannot fix the overall normalization of the measure, since this corresponds to adding a constant to the string field theory action, and the requirement that the action satisfies the BV master equation does not fix this constant.

\section{3 'Gauge invariant' path integral}

Let us now focus on the specific case of $(1,1)$ D-instanton in two dimensional string theory. In this case we have [28]:

$$
\mathcal{A}=\int_{0}^{\infty} \frac{d t}{2 t}\left(e^{2 \pi t}-1\right)
$$


Comparing this with (2.3) we see that the contribution from all states with $L_{0}>0$ cancel between bosonic and fermionic states. It follows that in the path integral expression (2.7) for $\mathbf{N}$, we can drop the integration over all states with $L_{0}>0$. Therefore we shall introduce a restricted string field $\left|\Psi_{R}\right\rangle$ given by a linear combination of basis states with $L_{0} \leq 0$. Before gauge fixing, $\left|\Psi_{R}\right\rangle$ has the following expansion:

$$
\begin{aligned}
\left|\Psi_{R}\right\rangle= & \psi^{0} c_{1}|0\rangle+\psi_{0} c_{0} c_{1}|0\rangle+\psi^{1} c_{0}|0\rangle+\psi^{2}|0\rangle+\psi_{1} c_{-1} c_{1}|0\rangle+\psi_{2} c_{-1} c_{0} c_{1}|0\rangle \\
& +\psi^{3} c_{1} \alpha_{-1}|0\rangle+\psi_{3} c_{0} c_{1} \alpha_{-1}|0\rangle
\end{aligned}
$$

where $|0\rangle$ is the $\mathrm{SL}(2, \mathrm{R})$ invariant vacuum, $c_{n}, b_{n}$ are the usual ghost oscillators and $\alpha_{m}$ are the oscillators associated with the Euclidean time coordinate $X$, satisfying $\left[\alpha_{m}, \alpha_{n}\right]=$ $m \delta_{m+n, 0}$. In the $\alpha^{\prime}=1$ unit the $X$ 's satisfy the operator product expansion ${ }^{2}$

$$
\partial X(z) \partial X(w)=-\frac{1}{2(z-w)^{2}} .
$$

This leads to the following state operator correspondence:

$$
c_{1} \alpha_{-1}|0\rangle=i \sqrt{2} c(0) \partial X(0)|0\rangle .
$$

The basis states in which we have expanded the string field in (3.2) are normalized according to (2.10) provided we choose:

$$
\left\langle 0\left|c_{-1} c_{0} c_{1}\right| 0\right\rangle=1
$$

In this case the $\left\{\psi^{r}\right\}$ 's label fields and the $\left\{\psi_{r}\right\}$ 's label the conjugate anti-fields in the BV formalism.

In the Siegel gauge, the modes that survive are $\psi^{0}, \psi_{1}, \psi^{2}$ and $\psi^{3}$. Of these, $\psi^{0}$ and $\psi^{3}$ are bosonic modes and $\psi_{1}$ and $\psi^{2}$ are fermionic modes. Therefore, (2.7) may now be written as:

$$
\mathbf{N}=\zeta \int \frac{d \psi^{0}}{\sqrt{2 \pi}} \int \frac{d \psi^{3}}{\sqrt{2 \pi}} d \psi_{1} d \psi^{2} e^{S}
$$

However, as discussed in $[11,13]$, this is a singular gauge choice due to the presence of the zero mode $\psi_{1}$. We avoid this problem by choosing the 'gauge' in which $\psi_{1}=0$ but $\psi^{1} \neq 0$. In this case all the anti-fields are set to zero and we integrate over all the field modes $\psi^{0}, \psi^{1}, \psi^{2}, \psi^{3}{ }^{3}$ Now we have three bosonic modes $\psi^{0}, \psi^{1}, \psi^{3}$ and one fermionic mode $\psi^{2}$. The action (2.9) now takes the form:

$$
S=-\left[-\frac{1}{2}\left(\psi^{0}\right)^{2}-\left(\psi^{1}\right)^{2}\right]
$$

This shows that we still have a pair of zero modes — one bosonic zero mode $\psi^{3}$ and one fermionic zero mode $\psi^{2}$ over which we need to integrate. Since $\psi^{2}$ is a grassmann odd variable, naively the integral would vanish. However, the mode $\psi^{2}$ is the ghost field associated

\footnotetext{
${ }^{2}$ We shall use the standard doubling trick in which we regard $\partial X$ as an analytic function over the full complex plane, with the understanding that $\partial X(z)$ for $z$ in the lower half plane actually represents $-\bar{\partial} X(z)$.

${ }^{3}$ Consequences of this for tree amplitudes have been discussed in [11, 29, 30].
} 
with the string field theory gauge transformation generated by $\theta|0\rangle$ for a parameter $\theta$, and the integration over $\psi^{2}$ can be interpreted as division by $\int d \theta$, with the integral running over the volume of the gauge group $[11,13]$. This allows us to express (3.6) as,

$$
\mathbf{N}=\zeta \int \frac{d \psi^{0}}{\sqrt{2 \pi}} \int \frac{d \psi^{3}}{\sqrt{2 \pi}} d \psi^{1} e^{S} / \int d \theta
$$

In the BV formalism the equivalence of the 'gauge invariant' form of the path integral, where we set all the anti-fields to zero, to the Siegel gauge fixed version, is usually proved at the level of correlation functions [18-20] for which the overall normalization of the path integral cancels. Since the normalization is important for us, we have shown in appendix A that the equality of (3.6) and (3.8) can be understood using the standard Faddeev-Popov formalism.

We shall now show that (3.8) leads to finite unambiguous result. Let us first carry out the integral over $\psi^{0}$ and $\psi^{1}$ by taking the integration contours to be the steepest descent contours. Both of these lie along the imaginary axis, and the final result takes the form:

$$
\mathbf{N}=-\zeta \frac{1}{\sqrt{2}} \int d \psi^{3} / \int d \theta
$$

The minus sign in (3.9) is the result of the product of two $i$ 's, one from having to integrate the tachyon $\psi^{0}$ along the imaginary axis and the other from having to integrate $\psi^{1}$ along the imaginary axis. However in the open string field theory, the reality condition on the mode $\psi^{1}$ is $\left(\psi^{1}\right)^{*}=-\psi^{1}$ [31], indicating that we should carry out the path integral over the variable $i \psi^{1}$ instead of $\psi^{1}$. This would remove one factor of $i$ from (3.9). There is however a similar factor of $i$ involved in the integration over the gauge transformation parameter $\theta$ in (3.8). These effects cancel each other, and so we shall proceed with (3.9) without removing any factor of $i$. This has been discussed in footnote 4 .

The mode $\psi^{3}$ is related by field redefinition to the collective mode corresponding to the freedom of translating the D-instanton along the Euclidean time direction. If $\widetilde{\phi}$ denotes the correctly normalized collective mode that measures the amount of translation along the time coordinate, then the dependence of any amplitude on $\widetilde{\phi}$ should be of the form $e^{-i \omega \widetilde{\phi}}$ where $\omega$ is the total energy carried by all the external closed string states. Therefore the relation between $\psi^{3}$ and $\widetilde{\phi}$ may be found by studying the coupling of $\psi^{3}$ to an amplitude and comparing this with the expected coupling of $\widetilde{\phi}$ to the same amplitude. Let us begin with a disk amplitude of a set of closed string states carrying energies $\omega_{1}, \omega_{2}, \cdots$. Since the vertex operator of the state associated with $\psi^{3}$ is given by $i \sqrt{2} c \partial X$, inserting this into this amplitude will correspond to inserting the integrated vertex operator

$$
i \sqrt{2} \int \partial X(z) d z
$$

Using the operator product expansion (3.3), and recalling that when we use the doubling trick mentioned in footnote 2 , insertion of a vertex operator $e^{-i \omega_{k} X\left(w_{k}\right)}$ is implicitly ac- 
companied by its image $e^{i \omega_{k} X\left(\bar{w}_{k}\right)}$, we get

$$
\begin{aligned}
\langle i & \left.\sqrt{2} \int \partial X(z) d z \prod_{k} e^{-i \omega_{k} X\left(w_{k}\right)}\right\rangle \\
& =i \sqrt{2} \sum_{j} \int d z\left[\left\{\frac{i \omega_{j}}{2\left(z-w_{j}\right)}-\frac{i \omega_{j}}{2\left(z-\bar{w}_{j}\right)}\right\}\left\langle\prod_{k} e^{-i \omega_{k} X\left(w_{k}\right)}\right\rangle\right] \\
& =-2 \pi i \frac{\omega}{\sqrt{2}}\left\langle\prod_{k} e^{-i \omega_{k} X\left(w_{k}\right)}\right\rangle, \quad \omega \equiv \sum_{j} \omega_{j} .
\end{aligned}
$$

Since we have not included any dependence on the string coupling in the quadratic terms in the action, the open string vertex operator (3.10) should also carry a factor of the open string coupling $g_{o} \propto \sqrt{g_{s}}$. The precise relation between $g_{o}$ and $g_{s}$ was determined in [25] and takes the form $g_{o}^{2}=g_{s} /\left(2 \pi^{2}\right)$ in the convention in which the instanton action is given by $1 / g_{s}$. We shall proceed for now by ignoring the factors of $g_{o}$ since $g_{o}$ (in)dependence of $\mathbf{N}$ has already been understood in [32]. At the end of this section we shall briefly discuss $g_{o}$ dependence of different contributions to $\mathbf{N}$ and show how they cancel. Eq. (3.11) now shows that coupling of $\psi^{3}$ to an amplitude with closed string states carrying total energy $\omega$ generates a factor of $-\sqrt{2} \pi i \omega$. On the other hand, since the dependence of an amplitude on the collective coordinate $\widetilde{\phi}$ is of the form $e^{-i \omega \widetilde{\phi}}=(1-i \omega \widetilde{\phi}+\cdots)$, the coupling of $\widetilde{\phi}$ to an amplitude with closed string state carrying energy $\omega$ generates a factor of $-i \omega$. This gives the identification of $-\sqrt{2} \pi i \omega \psi^{3}$ to $-i \omega \widetilde{\phi}$, in agreement with the results of [25]. Therefore in (3.9) we can make the replacement:

$$
d \psi^{3}=\frac{1}{\sqrt{2} \pi} d \widetilde{\phi}
$$

Integration over the collective mode $\widetilde{\phi}$ generates the usual energy conserving delta function $2 \pi \delta(\omega)$ that is part of any amplitude and is not included in the normalization constant $\mathbf{N}$. Therefore we can now express (3.9) as

$$
\mathbf{N}=-\zeta \frac{1}{\sqrt{2}} \frac{1}{\sqrt{2} \pi} / \int d \theta=-\zeta \frac{1}{2 \pi} / \int d \theta .
$$

We now turn to the evaluation of $\int d \theta$. Physically, this gauge transformation is related by field redefinition to the rigid $\mathrm{U}(1)$ gauge transformation that multiplies any state of the open string, stretched from the D-instanton to a second D-instanton, by $e^{i \widetilde{\theta}}$. Since $\tilde{\theta}$ has period $2 \pi$, in order to determine the range of $\theta$ integral, we need to find the relation between $\theta$ and $\widetilde{\theta}$. This in turn can be determined by comparing the string field theory gauge transformation law generated by $\theta$ to the rigid $\mathrm{U}(1)$ gauge transformation with parameter $\tilde{\theta}$ for any state of the open string that connects the D-instanton to the second D-instanton. This is achieved as follows:

1. As in [13], we shall work with a particular mode $\xi$ that multiplies the vacuum state $|0\rangle$ of the open string stretched between the two D-instantons but the relation between $\theta$ and $\widetilde{\theta}$ is independent of this choice. The conjugate anti-field $\xi^{*}$ of $\xi$ will multiply 
the state $c_{-1} c_{0} c_{1}|0\rangle$ of the open string that connects the second D-instanton to the original D-instanton.

2. The vertex operators associated with the modes $\xi$ and $\xi^{*}$ are accompanied by ChanPaton factors $\left(\begin{array}{ll}0 & 1 \\ 0 & 0\end{array}\right)$ and $\left(\begin{array}{ll}0 & 0 \\ 1 & 0\end{array}\right)$ respectively, and the open string mode $\psi^{2}$, that connects the original D-instanton to itself, carries Chan-Paton factor $\left(\begin{array}{ll}1 & 0 \\ 0 & 0\end{array}\right)$.

3. It follows from the gauge transformation laws of the string field theory that the gauge transformation of $\xi$ under the gauge transformation generated by $\theta$ is given by the second derivative of the action with respect to $\xi^{*}$ and $\psi^{2}$. The leading contribution comes from the $\xi-\xi^{*}-\psi^{2}$ coupling in the action arising from the disk amplitude. Since two of the three vertex operators - those associated with $\xi$ and $\psi^{2}$ are just identity operators, the coefficient of this term is given by

$$
\left\langle 0\left|c_{-1} c_{0} c_{1}\right| 0\right\rangle \operatorname{Tr}\left[\left(\begin{array}{ll}
0 & 1 \\
0 & 0
\end{array}\right)\left(\begin{array}{ll}
0 & 0 \\
1 & 0
\end{array}\right)\left(\begin{array}{ll}
1 & 0 \\
0 & 0
\end{array}\right)\right]=1 .
$$

This corresponds to the presence of a term

$$
\xi \xi^{*} \psi^{2}
$$

in the action if we ignore the factors of $g_{o}$ as before.

4. Taking the derivative of $(3.15)$ with respect to $\xi^{*}$ and $\psi^{2}$, we see that the gauge transformation generated by the parameter $\theta$ takes the form $\delta \xi=\theta \xi$. Comparing this with the infinitesimal rigid $\mathrm{U}(1)$ transformation $\delta \xi=i \widetilde{\theta} \xi$. we get $\theta=i \widetilde{\theta}{ }^{4}$

This gives

$$
\mathbf{N}=-\zeta \frac{1}{2 \pi} / \int d \theta=\zeta \frac{i}{2 \pi} / \int d \widetilde{\theta}=\zeta \frac{i}{4 \pi^{2}}
$$

Finally we shall discuss the dependence of $\mathbf{N}$ on the string coupling. This has already been fully understood in [32] but we include the discussion here for completeness. We denote by $g_{o}=\sqrt{g_{s} /\left(2 \pi^{2}\right)}$ the open string coupling [25]. We shall work in the convention in which the kinetic term of the open string fields has $g_{o}$ independent normalization, so that in the Siegel gauge the quadratic part of the action is $g_{o}$ independent, in agreement with the $g_{o}$ independent exponent appearing in (2.7). In this convention, each open string vertex operator carries a factor of $g_{o}$. This introduces an additional factor of $g_{o}$ in (3.10), (3.11) and therefore a factor of $1 / g_{o}$ in the right hand sides of (3.12), (3.13), (3.16). On the other hand the disk three point function of three open string vertex operators now gets a factor of $g_{o}^{-2}$ from the disk, and a factor of $g_{o}^{3}$ from the three open string vertex operators, producing a net factor of $g_{o}$. Therefore (3.15) gets a factor of $g_{o}$, leading to the gauge

\footnotetext{
${ }^{4}$ This factor of $i$ is the result of imposing wrong reality condition on the mode $\psi^{2}$ or equivalently the parameter $\theta$. We have not corrected it since this cancels the factor of $i$ arising out of the wrong choice of reality condition for the mode $\psi^{1}$. This has been discussed below (3.9).
} 


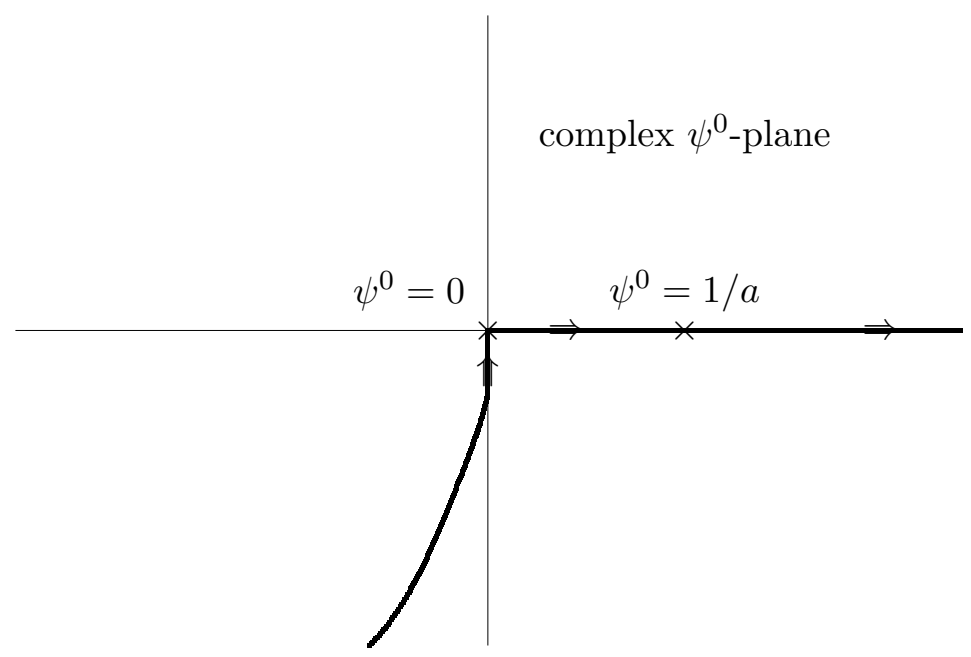

Figure 1. The integration contour in the complex $\psi^{0}$ plane.

transformation law $\delta \xi=g_{o} \theta \xi$. Therefore we now have $g_{o} \theta=i \widetilde{\theta}$, leading to an extra factor of $g_{o}$ on the right hand side of (3.16). This cancels the earlier factor of $1 / g_{o}$ and leaves the right hand side of (3.16) unchanged. Therefore $\mathbf{N}$ is $g_{o}$ independent.

\section{The multiplier}

We now turn to the determination of the multiplier $\zeta$. For this we need to know how the steepest descent contour / Lefschetz thimble passing through the saddle point representing the D-instanton fits inside the actual integration cycle that computes the full amplitude in string theory [22-24]. For the case of two dimensional bosonic string theory this was discussed in [27] where it was argued that the actual integration contour contains only half of this thimble. In brief, the argument can be stated as follows. After integrating out the massive open string modes, the tachyon effective potential on the D-instanton has a potential $V\left(\psi^{0}\right)$ that has a maximum at $\psi^{0}=0$ describing the D-instanton and a minimum at some positive value $1 / a$ describing the perturbative vacuum where the potential vanishes. The potential is unbounded from below as $\psi^{0} \rightarrow-\infty$. Therefore the integration contour over $\psi^{0}$ cannot be taken to be along the real axis all the way to $-\infty$, but near the perturbative vacuum where the potential has a local minimum we expect the contour to lie along the real axis. If we model the potential as

$$
V\left(\psi^{0}\right)=-\frac{1}{2}\left(\psi^{0}\right)^{2}+\frac{1}{3} a\left(\psi^{0}\right)^{3}+\frac{1}{6 a^{2}},
$$

then one can easily see that the potential goes to $+\infty$ as we approach the asymptotic region within three $60^{\circ}$ cones, centered around the lines $\psi^{0}=r, \psi^{0}=r e^{2 \pi i / 3}$ and $\psi^{0}=r e^{-2 \pi i / 3}$ for real positive $r$. Therefore we can take the integration contour to interpolate between the regions $\psi^{0}=e^{-2 \pi i / 3} \times \infty$ and $\psi^{0}=\infty$ as shown in figure 1 or we can choose the complex conjugate contour. On the other hand, the steepest descent contour for the saddle point at $\psi^{0}=1 / a$, representing the perturbative vacuum, lies along the real $\psi^{0}$ axis from 
0 to $\infty$, while the steepest descent contour for the saddle point at $\psi^{0}=0$, representing the D-instanton, consists of a contour through the origin that interpolates between the regions around $\psi^{0}=e^{-2 \pi i / 3} \times \infty$ and $\psi^{0}=e^{2 \pi i / 3} \times \infty$. Therefore the integration contour shown in figure 1 can be regarded as the union of the steepest descent contour of the saddle point at $\psi^{0}=a$, and half of the steepest descent contour of the saddle point at $\psi^{0}=0$. This gives

$$
\zeta=\frac{1}{2}
$$

and

$$
\mathbf{N}=\frac{i}{8 \pi^{2}}
$$

Let us now comment on the sign of $\mathbf{N}$ about which we have not been careful so far. This clearly depends on the choice of the full integration contour - if instead of the contour shown in figure 1 we choose the complex conjugate contour, the sign of $\mathbf{N}$ will change. The actual choice should be dictated by physical considerations, e.g. if a Dinstanton induced amplitude leads to violation of unitarity, it should be describable by an effective Hamiltonian with negative imaginary part, reflecting loss of probability due to possible transition to states that have not been accounted for in the effective Hamiltonian. As discussed in [27], the choice of sign given in (4.3) is the correct choice according to this consideration. Therefore (4.3) gives the final result for the normalization constant associated with single D-instanton amplitudes in two dimensional string theory. This agrees with the result obtained in [3] by comparison with the matrix model results for the instanton induced amplitudes, after we multiply this by a factor of $i$ to compute the normalization of the D-instanton contribution to the S-matrix elements.

\section{Discussion}

The method described here can in principle be applied to other D-instanton systems, e.g. general $(m, n)$ ZZ-instantons in two dimensional bosonic string theory [28], D-instantons in two dimensional type 0B string theory $[33,34]$ and D-instantons in type IIB string theory $[2,35]$. Part of the analysis that may be somewhat non-trivial is the computation of the multiplier factor $\zeta$, since this requires the knowledge of how the steepest descent contour / Lefschetz thimble associated with a particular D-instanton fits into the full integration contour. For example, in the context of $(m, n)$ ZZ-instantons in two dimensional string theory, this will require the knowledge of how the different ZZ-instantons are represented as different extrema in the configuration space of string fields. However since the multiplier factors are just rational numbers, we only need topological information on the locations of various extrema in the configuration space of string fields instead of requiring detailed dynamical information. Therefore we do not consider this to be an insurmountable problem. Similarly for computing the D-instanton contribution to the type IIB string theory amplitude, we need to understand how the D-instantons, which in this case represent complex saddle points, fit inside the integration contour over the string fields. 


\section{Acknowledgments}

I wish to thank Bruno Balthazar, Victor Rodriguez, Xi Yin and Barton Zwiebach for many useful discussions. This work was supported in part by the J. C. Bose fellowship of the Department of Science and Technology, India and the Infosys chair professorship.

\section{A Siegel gauge fixing in the Faddeev-Popov formalism}

In our analysis the formal equality between the Siegel gauge fixed path integral (3.6) and the gauge invariant path integral (3.8) plays an important role. In this appendix we shall show how this can be proved using the standard Faddeev-Popov formalism.

Classical open string field $\left|\psi_{c}\right\rangle$ is an arbitrary state of ghost number 1 of the open string and the gauge transformation parameters describing the symmetries of the classical open string field theory correspond to an arbitrary state $|\theta\rangle$ of ghost number 0. As in (3.2), we shall work with the restricted string field carrying $L_{0} \leq 0$. In this case, it is evident from (3.2) that the gauge transformation parameter $|\theta\rangle$, carrying ghost number 0 , satisfies the condition $b_{0}|\theta\rangle=0$. Let us introduce basis states $\left\{\left|\phi_{r}^{(n)}\right\rangle\right\}$ of ghost number $n$, satisfying $b_{0}\left|\phi_{r}^{(n)}\right\rangle=0$. Then the full set of basis states at ghost number $n$ may be taken to be $\left\{\left|\phi_{r}^{(n)}\right\rangle\right\}$ and $\left\{c_{0}\left|\phi_{s}^{(n-1)}\right\rangle\right\}$. We shall further normalize these basis states for $n=0$ and 2 such that

$$
\left\langle\phi_{r}^{(2)}\left|c_{0}\right| \phi_{s}^{(0)}\right\rangle=\delta_{r s} .
$$

We can now expand the restricted classical string field $\left|\psi_{c}\right\rangle$ and the gauge transformation parameter $|\theta\rangle$ as:

$$
\begin{aligned}
\left|\psi_{c}\right\rangle & =\sum_{r} \widetilde{\psi}_{r}\left|\phi_{r}^{(1)}\right\rangle+\sum_{s} \widehat{\psi}_{s} c_{0}\left|\phi_{s}^{(0)}\right\rangle, \\
|\theta\rangle & =\sum_{s} \theta_{s}\left|\phi_{s}^{(0)}\right\rangle .
\end{aligned}
$$

The gauge invariant classical action up to quadratic order and the linearized gauge transformation laws are given, respectively, by,

$$
S_{g . i .}=-\frac{1}{2}\left\langle\psi_{c}\left|Q_{B}\right| \psi_{c}\right\rangle
$$

and

$$
\delta\left|\psi_{c}\right\rangle=Q_{B}|\theta\rangle
$$

We now consider a gauge invariant path integral of the form:

$$
I_{g . i .}=\int \prod_{r} d \widetilde{\psi}_{r} \prod_{s} d \widehat{\psi}_{s} e^{S_{g . i .}} / \int \prod_{s} d \theta_{s}
$$

We can evaluate this by gauge fixing in the Siegel gauge, by setting $\widehat{\psi}_{s}=0$. This introduces a factor of $\prod_{s} \delta\left(\widehat{\psi}_{s}\right)$ in the path integral, accompanied by the appropriate determinant. To 
find the determinant we need to examine the gauge transformation law of $\widehat{\psi}_{s}$. Substituting (A.2), (A.3) into (A.5) and taking the inner product of the resulting equation with $\left\langle\phi_{s}^{(2)}\right|$, we get

$$
\delta \widehat{\psi}_{s}=\left\langle\phi_{s}^{(2)}\left|Q_{B}\right| \theta\right\rangle=M_{s u} \theta_{u}, \quad M_{s u}=\left\langle\phi_{s}^{(2)}\left|Q_{B}\right| \phi_{u}^{(0)}\right\rangle .
$$

The determinant entering the integrand of the path integral is det $M$. We can express this as a path integral over a pair of grassmann odd ghost variables $\left\{p_{s}\right\},\left\{q_{s}\right\}$. This gives the gauge fixed path integral:

$$
I_{g . f .}=\left.\int \prod_{r} d \psi_{r} \prod_{s} d p_{s} d q_{s} e^{S_{g . i .}+S_{\text {ghost }}}\right|_{\widehat{\psi}_{s}=0}, \quad S_{\text {ghost }}=-\sum_{s, u} p_{s} M_{s u} q_{u}=-\left\langle P\left|Q_{B}\right| Q\right\rangle,
$$

where we have introduced

$$
|P\rangle=\sum_{s} p_{s}\left|\phi_{s}^{(2)}\right\rangle, \quad|Q\rangle=\sum_{s} q_{s}\left|\phi_{s}^{(0)}\right\rangle .
$$

The equality of $I_{g . i .}$ and $I_{g . f}$. give in (A.6) and (A.8) in the context of two dimensional string theory gives the equality of (3.8) and (3.6). To see this note that in this case the master field $|\psi\rangle$ contains fields of all ghost number without any gauge condition, and the master action is $-\frac{1}{2}\left\langle\psi\left|Q_{B}\right| \psi\right\rangle$. When we pick the Lagrangian submanifold in which we set the modes of ghost number $\geq 2$ to 0 , the master action reduces to (A.4) and (3.8) may be interpreted as $\zeta /(2 \pi)$ times $I_{g . i .}$ given in (A.6). On the other hand when we pick the Lagrangian submanifold by imposing the Siegel gauge condition, the master action reduces to $S_{g . i .}+S_{\text {ghost }}$ given in (A.8) and (3.6) may be interpreted as $\zeta /(2 \pi)$ times $I_{g . f}$. given in (A.8).

The equality of $I_{g . i .}$ and $I_{g . f}$. that we have established is formal, since in the context in which we apply this, there are component fields that multiply basis states of vanishing $L_{0}$ eigenvalue. This means that the Siegel gauge action becomes independent of those fields, making the path integral (A.8) ill-defined. This can be traced to the fact that the Siegel gauge $\widehat{\psi}_{r}=0$ is a singular gauge choice due to the appearance of zero eigenvalues of the matrix $M$ introduced in (A.7). However the original gauge invariant path integral (A.6) is well defined. The point of view we take is that (A.6) is the proper definition of the path integral, and the problem we face with (A.8) arising in the world-sheet formalism is due to illegal choice of gauge. ${ }^{5}$

The analysis described above also resolves an apparent puzzle that arises out of the equality of (3.6) and (3.8). Since the integral in (3.6) has equal number of bosonic and fermionic variables, it remains unchanged if we multiply $S$ in the exponent by some constant $C$. Following the logic that led from (3.6) to (3.8), we can see that the effect of this rescaling is to multiply the exponent in (3.8) by the same constant $C$. Since in this expression both $\psi^{0}$ and $\psi^{1}$ represent bosonic variables, this will produce a factor of $1 / C$ on the right hand side of (3.9) that carries over to the right hand sides of (3.13) and (3.16). So the question is: what compensates this factor?

\footnotetext{
${ }^{5}$ As discussed below (2.7), $I_{g . f}$. can be made well-defined by working with open strings stretched between a pair of separated D-instantons. In that case the equality between $I_{g . i}$. and $I_{g . f}$. becomes an identity.
} 
The answer to this question comes from (A.7) and (A.8). If the exponent of (3.6) is multiplied by $C$, then the ghost action involving the modes $p_{s}, q_{s}$, which correspond to $\psi^{2}$ and $\psi_{1}$ in (3.6), also gets multiplied by $C$. Therefore the matrix $M$ in (A.7) must be multiplied by $C$. This can happen if we include an extra factor of $C$ in the gauge transformation law (A.5). If we denote by $\theta^{\prime}$ the new gauge transformation parameter, then it is related to the old gauge transformation parameter $\theta$ by $\theta=C \theta^{\prime}$. Therefore the $\int d \theta^{\prime}$ factor that now appears in the denominator can be identified to $\int d \theta / C$, and we get an extra multiplicative factor of $C$ on the right hand side of (3.9). This cancels the extra factor of $1 / C$ coming from the integration over $\psi^{0}$ and $\psi^{1}$.

Open Access. This article is distributed under the terms of the Creative Commons Attribution License (CC-BY 4.0), which permits any use, distribution and reproduction in any medium, provided the original author(s) and source are credited.

\section{References}

[1] J. Polchinski, Combinatorics of boundaries in string theory, Phys. Rev. D 50 (1994) R6041 [hep-th/9407031] [INSPIRE].

[2] M.B. Green and M. Gutperle, Effects of D instantons, Nucl. Phys. B 498 (1997) 195 [hep-th/9701093] [INSPIRE].

[3] B. Balthazar, V.A. Rodriguez and X. Yin, ZZ instantons and the non-perturbative dual of $c=1$ string theory, arXiv:1907.07688 [INSPIRE].

[4] E. Witten, Noncommutative geometry and string field theory, Nucl. Phys. B 268 (1986) 253 [INSPIRE].

[5] B. Zwiebach, Closed string field theory: quantum action and the B-V master equation, Nucl. Phys. B 390 (1993) 33 [hep-th/9206084] [INSPIRE].

[6] B. Zwiebach, Oriented open-closed string theory revisited, Annals Phys. 267 (1998) 193 [hep-th/9705241] [INSPIRE].

[7] C. de Lacroix, H. Erbin, S.P. Kashyap, A. Sen and M. Verma, Closed superstring field theory and its applications, Int. J. Mod. Phys. A 32 (2017) 1730021 [arXiv:1703.06410] [InSPIRE].

[8] T. Erler, Four lectures on closed string field theory, Phys. Rept. 851 (2020) 1 [arXiv: 1905.06785] [INSPIRE].

[9] Y. Okawa, Complete formulation of superstring field theory, in Noncommutative geometry and physics 4, World Scientific, Singapore (2017), pg. 49.

[10] A. Sen, Fixing an ambiguity in two dimensional string theory using string field theory, JHEP 03 (2020) 005 [arXiv: 1908.02782] [INSPIRE].

[11] A. Sen, D-instanton perturbation theory, JHEP 08 (2020) 075 [arXiv:2002.04043] [INSPIRE].

[12] A. Sen, Divergent $\Longrightarrow$ complex amplitudes in two dimensional string theory, JHEP 02 (2021) 086 [arXiv: 2003.12076] [INSPIRE].

[13] A. Sen, D-instantons, string field theory and two dimensional string theory, arXiv:2012.11624 [INSPIRE].

[14] G.W. Moore, M.R. Plesser and S. Ramgoolam, Exact $S$ matrix for $2 D$ string theory, Nucl. Phys. B 377 (1992) 143 [hep-th/9111035] [InSPIRE]. 
[15] I.A. Batalin and G.A. Vilkovisky, Gauge algebra and quantization, Phys. Lett. B 102 (1981) 27 [INSPIRE].

[16] I.A. Batalin and G.A. Vilkovisky, Quantization of gauge theories with linearly dependent generators, Phys. Rev. D 28 (1983) 2567 [Erratum ibid. 30 (1984) 508] [INSPIRE].

[17] M. Henneaux and C. Teitelboim, Quantization of gauge systems, Princeton University Press, Princeton, NJ, U.S.A. (1992).

[18] M. Bochicchio, Gauge fixing for the field theory of the bosonic string, Phys. Lett. B 193 (1987) 31 [INSPIRE].

[19] M. Bochicchio, String field theory in the Siegel gauge, Phys. Lett. B 188 (1987) 330 [INSPIRE].

[20] C.B. Thorn, String field theory, Phys. Rept. 175 (1989) 1 [InSPIRE].

[21] A.S. Schwarz, Geometry of Batalin-Vilkovisky quantization, Commun. Math. Phys. 155 (1993) 249 [hep-th/9205088] [INSPIRE].

[22] M. Mariño, Lectures on non-perturbative effects in large $N$ gauge theories, matrix models and strings, Fortsch. Phys. 62 (2014) 455 [arXiv:1206.6272] [INSPIRE].

[23] G.V. Dunne and M. Ünsal, What is QFT? Resurgent trans-series, Lefschetz thimbles, and new exact saddles, PoS LATTICE2015 (2016) 010 [arXiv:1511.05977] [INSPIRE].

[24] I. Aniceto, G. Basar and R. Schiappa, A primer on resurgent transseries and their asymptotics, Phys. Rept. 809 (2019) 1 [arXiv:1802.10441] [INSPIRE].

[25] A. Sen, Universality of the tachyon potential, JHEP 12 (1999) 027 [hep-th/9911116] [INSPIRE].

[26] M. Kiermaier, Y. Okawa and B. Zwiebach, The boundary state from open string fields, arXiv: 0810.1737 [INSPIRE].

[27] A. Sen, Cutkosky rules and unitarity (violation) in D-instanton amplitudes, arXiv:2012.00041 [INSPIRE].

[28] B. Balthazar, V.A. Rodriguez and X. Yin, Multi-instanton calculus in $c=1$ string theory, arXiv: 1912.07170 [INSPIRE].

[29] H. Erbin, C. Maccaferri and J. Vošmera, Localization of effective actions in heterotic string field theory, JHEP 02 (2020) 059 [arXiv: 1912.05463] [INSPIRE].

[30] H. Erbin, C. Maccaferri, M. Schnabl and J. Vošmera, Classical algebraic structures in string theory effective actions, JHEP 11 (2020) 123 [arXiv:2006.16270] [INSPIRE].

[31] M.R. Gaberdiel and B. Zwiebach, Tensor constructions of open string theories. 1: foundations, Nucl. Phys. B 505 (1997) 569 [hep-th/9705038] [InSPIRE].

[32] B. Balthazar, V.A. Rodriguez and X. Yin, private communication.

[33] T. Takayanagi and N. Toumbas, A matrix model dual of type $0 B$ string theory in two-dimensions, JHEP 07 (2003) 064 [hep-th/0307083] [INSPIRE].

[34] M.R. Douglas, I.R. Klebanov, D. Kutasov, J.M. Maldacena, E.J. Martinec and N. Seiberg, $A$ new hat for the $c=1$ matrix model, in From fields to strings: circumnavigating theoretical physics. A conference in tribute to Ian Kogan, (2003), pg. 1758 [hep-th/0307195] [INSPIRE].

[35] M. Gutperle, Aspects of D instantons, NATO Sci. Ser. C 520 (1999) 411 [hep-th/9712156] [INSPIRE]. 